\title{
DIVERSITY, DIETS AND DISPARITY: DETERMINING THE EFFECT OF THE TERMINAL CRETACEOUS EXTINCTION ON INSECT EVOLUTION
}

\section{LABANDEIRA, Conrad C., Department of Paleobiology, National Museum of Natural History, Smithsonian Institution, Washington D.C., 20560}

A considerable amount of research has been devoted toward evaluating the impact of the Cretaceous/Tertiary extinction on terrestrial life. This research has focused primarily on terrestrial vertebrates (primarily dinosaurs), marine invertebrates (notably molluscs and foraminifera), and to a lesser extent, terrestrial vascular plants. Terrestrial arthropods, especially insects, have seldomly been investigated, principally because of an alleged depauperate fossil record. Nevertheless, within the past two decades, some of the most productive and taxonomically diverse insect faunas have originated from Cretaceous amber- and compression-fossil deposits from every continent. Whereas it was once thought that the Cretaceous represented an unknown void in the understanding of insect evolution, now it appears that many extant lineages are traceable to Cretaceous precursors.

Three approaches aro available for determining the extent of the effect of the terminal Cretaceous extinction event on insects. Assessed for the interval from the Early Cretaceous to the Early Paleogene, these approaches are: (1) establishing the secular pattern of familial- and generic-based taxonomic diversity (macroevolution); (2) recognizing the persistence or eradication of specific insect/vascular plant interactions, such as leafmining, wood-boring and pollination (behavior); and (3) establishing temporal trends in the range of mouthpart design, as an indicator of faunal disparity or structural diversity (morphology). These three operationally separate but complimentary approaches allow the advantage of using distinct data bases to bear on a common question. The bodyfossil record of insects provides primary data for the taxonomic expansion, steady-state, or contraction of insect faunas. The trace-fossil record of those insect interactions that are coevolved with plant hosts reveals the temporal continuity of highly stereotyped and taxonomically obligate behaviors. Both of these are contrasted to an assessment of insectan structural disparity, herein determined from a robust data base of 30 modern insect mouthpart classes that are traced back in geologic time.

A preliminary analysis of each of these three approaches indicates broad agreement-namely that insects were not dramatically affected by the terminal Cretaceous extinction event. First, insects experienced only a modest decline in diversity, about 9 percent at the family level. (The generic level is not yet analyzed.) Second, although the data base is limited, there is no indication of the extinction of major leaf-mining, wood-boring, pollinating or other plant-specific behaviors at the end of the Cretaceous. In fact, leafmine morphologies for three lepidopteran families with Cretaceous occurrences are apparently indistinguishable from their modern descendants. Last, of the 30 mouthpart classes occurring during the Paleogene, 28 are represented during the Cretaceous. These data provide strong evidence for a largely uninterrupted continuum of insect faunas across the Cretaceous/Tertiary boundary as measured by taxonomic diversity, coevolved behavior, and structural disparity.

Because of abundant and often intimate associations between insects and flowering plants, these results are consistent with a gradual and not catastrophic change in terrestrial floras across the Cretaceous/Tertiary boundary. Acceptance of a catastrophic extinction of flowering plants during the terminal Cretaceous would necessitate an unprecedented level of host-switching by coevolved insects on contemporaneous plants. This is unlikely, based on evidence from the prolific literature on modern insect/plant interactions. These studies indicate the ubiquity of obligate insect specificity for various secondary chemicals on many flowering plant species. 